\title{
Plattform-Regulierung. Koordination von Märkten und Kuratierung von Sozialität im Internet
}

\author{
Ulrich Dolata
}

Online publiziert: 28. April 2020

(C) Der/die Autor(en) 2020

Zusammenfassung Die führenden Internetkonzerne sind mit ihren weitläufig vernetzten Plattformen im Laufe der 2010er-Jahre zu den entscheidenden Akteuren der Gestaltung und regulativen Einfassung des Internets geworden. Der Aufsatz geht der Frage nach, über welche Ansatzpunkte und Mechanismen sie ihre Rolle als strukturbildende, regelsetzende und handlungskoordinierende Kernakteure im heutigen Web ausfüllen. Im Zentrum stehen dabei zwei wesentliche Regelungsbereiche: zum einen die privatwirtschaftliche Organisierung und Regulierung von Märkten, auf denen sie als Plattformbetreiber selbst die Marktprozesse koordinieren und die Wettbewerbsbedingungen festlegen, und zum anderen die technisch vermittelte Strukturierung und Kuratierung sozialer Verhältnisse und sozialen Verhaltens, durch die die Plattformbetreiber sehr weitreichende soziale Ordnungs- und Regulierungsfunktionen übernehmen und die institutionellen Grundlagen für eine privatwirtschaftlich verfasste Gesellschaftlichkeit im Web schaffen. Die wenigen großen Plattformen, die heute weite Teile des privaten und öffentlichen Lebens im Internet ermöglichen und prägen, lassen sich - so die These des Aufsatzes - als ausdifferenzierte gesellschaftliche Strukturen mit distinkter institutioneller Basis fassen, die die Plattformbetreiber über eigene Regeln, Regulierungen und Koordinationsgremien maßgeblich prägen und kontrollieren. Dies reicht bis hin zur Übernahme bislang staatlichen Instanzen vorbehaltener quasi-hoheitlicher Aufgaben durch die Unternehmen, die sich demokratischer Legitimation und Kontrolle weitgehend entziehen können.

Schlüsselwörter Internet · Plattformen · Regulierung · Digitaler Kapitalismus · Techkonzerne $\cdot$ Kuratierung $\cdot$ Algorithmen 


\title{
Platform-regulation. Coordination of markets and curation of sociality on the Internet
}

\begin{abstract}
Over the course of the 2010s, the leading Internet groups, with their extensively interconnected platforms, have become the key players in the design and regulatory framing of the Internet. This paper examines the mechanisms by which they fulfil their role as structure-building, rule-making and action-coordinating core actors in today's Web. The focus is on two essential regulatory areas: on the one hand, the private-sector organization and regulation of markets, in which they themselves, as platform operators, coordinate market processes and determine the conditions of competition; on the other hand, the technically mediated structuration and curation of social relationships and social behavior, through which the platform operators assume far-reaching regulatory functions. The few large platforms that today enable and coordinate large parts of private and public life on the Internet can - according to the thesis of this article - be understood as differentiated societal structures with a distinct institutional basis, which the platform operators shape and control to a considerable extent by means of their own rules, regulations and coordination bodies. This goes as far as the companies - which so far have been largely able to elude democratic legitimation and control - taking over quasi-sovereign tasks previously reserved for state authorities.
\end{abstract}

Keywords Internet · Platforms · Regulation - Digital Capitalism · Techcompanies · Curation · Algorithms 


\section{La régulation par les plates-formes. Coordination des marchés et curation de la sociabilité sur Internet}

Résumé Avec leur vastes plates-formes connectées en réseau, les géants d'Internet sont devenus au cours des années 2010 des acteurs décisifs du développement et de la régulation d'Internet. Cet article étudie les leviers et les mécanismes par le biais desquels ils remplissent leur rôle d'acteurs clés dans la création de structures, la mise en place de règles et la coordination des actions sur le Web d'aujourd'hui. L'accent est mis sur deux grands domaines de régulation: d'une part, l'organisation et la régulation des marchés sur lesquels ils coordonnent eux-mêmes, en tant qu'opérateurs de plates-formes, les opérations de marché et fixent les conditions de la concurrence; d'autre part, la structuration et la curation par des moyens techniques des rapports sociaux et du comportement social permettant aux opérateurs de platesformes d'assurer des fonctions très étendues d'organisation et de régulation sociale ainsi que de créer les fondements institutionnels d'une sociabilité réglée par le secteur privé sur le Web. La thèse présentée dans cet article est que les quelques grandes plates-formes qui permettent et façonnent aujourd'hui une grande partie de la vie privée et publique sur Internet peuvent être appréhendées comme des structures sociales disposant d'une base institutionnelle distincte sur lesquelles les opérateurs de plates-formes exercent une influence et un contrôle décisifs par le biais de règles, de régulations et d'organes de coordination spécifiques. Cela va jusqu'à la prise en charge de missions quasi-régaliennes auparavant réservées aux instances étatiques par des entreprises qui peuvent largement se soustraire à la légitimation et au contrôle démocratiques.

Mots-clés Internet · Plates-formes · Régulation · Capitalisme numérique · Grandes entreprises de technologie $\cdot$ Curation $\cdot$ Algorithme

\section{Einleitung: Privatwirtschaftliche Landnahme des Internets}

Gegen Mitte der 1990er-Jahre, also etwa zu der Zeit, als die kommerzielle ErschlieBung des Internets einsetzte - markiert etwa durch die Gründungen von Amazon (*1994), Yahoo! (*1995) oder Google $(* 1997)$-, standen Vorstellungen eines freien, dezentralen und selbstregulierten, aber auch bereits marktliberal strukturierten Netzes, das weitgehend ohne politische bzw. staatliche Eingriffe auskommen sollte, als einflussreiches, auch die Politik prägendes Narrativ hoch im Kurs der (netz-)politischen Debatten in den Vereinigten Staaten. John Perry Barlow (1996), einer der Gründer der Electronic Frontier Foundation, hatte am Rande des Weltwirtschaftsforums in Davos im Februar 1996 eine mit bemerkenswertem Pathos unterlegte und einem unbestimmten „Wir" versehene Declaration of the Independence of Cyberspace formuliert, die eine dezidierte Selbstregulierung des Netzes mit einem radikalen Anti-Etatismus und einer Absage an jeglichen staatlichen Gestaltungsversuch verknüpfte:

We are creating a world that all may enter without privilege or prejudice accorded by race, economic power, military force, or station of birth. We are creating 
a world where anyone, anywhere may express his or her beliefs, no matter how singular, without fear of being coerced into silence or conformity.

Und:

Governments of the Industrial World, you weary giants of flesh and steel, I come from Cyberspace, the new home of Mind. On behalf of the future, I ask you of the past to leave us alone. You are not welcome among us. You have no sovereignty where we gather.

Eineinhalb Jahre zuvor, im August 1994, hatten Esther Dyson, George Gilder, George Keyworth und Alvin Toffler (1994) eine Magna Carta for the Knowledge Age vorgelegt, in der libertäre Freiheitsauffassungen - ,America, after all, remains a land of individual freedom, and this freedom clearly extends to cyberspace" - und die offene Gestaltbarkeit des Netzes stärker mit neoliberalen Marktvorstellungen und einer dem technologischen Fortschritt unterstellten deterministischen Wirkkraft in Richtung Entmonopolisierung und Dezentralisierung der Wirtschaft kombiniert wurden:

In Cyberspace itself, market after market is being transformed by technological progress from a ,natural monopoly“ to one in which competition is the rule. [...] The advent of new technology and new products creates the potential for dynamic competition [...].

Diese Mischung aus freiheitlich-emanzipatorischen Gestaltungsvisionen, neoliberalen Marktauffassungen und technikdeterministischen Setzungen, die für die dann so bezeichnete ,kalifornische Ideologie“ typisch wurde, erwies sich in den folgenden Jahrzehnten als ausgesprochen wirkmächtiges Narrativ - auch weil sie die Weltsichten von zwei recht unterschiedlichen Akteurgruppen zusammenbringen konnte: Sie passte sowohl zum ,freewheeling spirit of the hippies“ als auch zum ,entrepreneurial zeal of the yuppies“ (Barbrook und Cameron 1996, S. 45). Später wurden diese Visionen sekundiert etwa von Vorstellungen einer ebenfalls aus neuen technischen Möglichkeiten hergeleiteten Handlungssouveränität und Gestaltungsfähigkeit der Nutzer im Web 2.0 (O'Reilly 2005; kritisch rekonstruierend: Schrape 2019).

Wesentliche Elemente der kalifornischen Ideologie und ihrer Nachfolger beruhten allerdings auf schon damals einer kritischen Beurteilung nicht standhaltendem „storytelling“. So wurde mit der Zurückweisung politischer Gestaltungs- und Regulierungsambitionen die keineswegs marginale Rolle des Staates im gesamten Entstehungs- und Entwicklungsprozess vernetzter Computersysteme und des Internets camoufliert. Vor allem die über Jahrzehnte und bis in die jüngste Vergangenheit betriebene intensive Forschungsförderung und -koordination des US-amerikanischen Staates, die zunächst vor allem aus dem Department of Defense und der dort angesiedelten Defense Advanced Research Projects Agency (DARPA) kam und später um spezifische technologie- und industriepolitische Förderprogramme etwa für StartupFirmen erweitert wurde, hat die Forschungs- und Innovationsdynamik sowie den akademisch-industriellen Wissenstransfer bis heute entscheidend mitgeprägt (Abbate 1999; Mazzucato 2013). Die Zurückweisung politischer Eingriffe zielte denn auch weniger auf die staatliche Forschungs(förder)politik, von der vor allem die Internet- 
unternehmen immer wieder massiv profitiert haben, als auf regulative Interventionen des Staates in das freie Spiel der (Markt-)Kräfte.

In place of counterproductive regulations, visionary engineers are inventing the tools needed to create a „free market" within cyberspace, such as encryption, digital money, and verification processes. (Barbrook und Cameron 1996, S. 53)

Auch das unspezifisch vorgetragene „Wir“ und mit ihm das Versprechen einer offenen und für jeden möglichen Gestaltbarkeit des Netzes war schon damals kaum mehr als haltlose Ideologie. Ende der 1990er-Jahre hatte Lawrence Lessig (1999) seine berühmte Metapher ,code is law“ formuliert und damit betont, dass das Web keineswegs ein regulierungsfreier Raum sei. Es sei allerdings weniger durch politische und rechtliche Regulierungen als durch komplexe informationstechnische Architekturen, Codes und Softwareanwendungen geprägt, in die immer soziale Setzungen und Anweisungen eingeschrieben sind, die auf zum Teil rigidere Weise als das Recht handlungsstrukturierend auf das Nutzerverhalten wirken (Feick und Werle 2010). Das „Wir“ tatsächlich substanziell gestaltungsfähiger Netzakteure schrumpfte damit auf eine kleine technisch versierte Elite derjenigen zusammen, die in der Lage waren und über die Ressourcen verfügten, entsprechende technische Spezifizierungen zu entwickeln, zu implementieren und zu kontrollieren.

Spätestens in den 2010er-Jahren ließ sich schließlich auch die Vision einer dezentral strukturierten Internetwirtschaft mit freien Märkten und vollständiger Konkurrenz nicht mehr halten. Im Schatten der lange sehr populären Vorstellungen einer offenen und staatsfernen Selbstorganisierbarkeit hatte die maßgeblich von Unternehmen aus dem Umfeld des Silicon Valley betriebene kommerzielle Erkundung und privat-regulative Strukturierung des Internets weitgehend unbehelligt von gesellschaftlichen Interventionen und staatlich-regulativen Einfassungen Fahrt auf- und Gestalt angenommen (Misterek 2017). Massive Konzentrationsprozesse, die Herausbildung von Winner-take-all-Märkten und die Etablierung neuer natürlicher QuasiMonopole, die das Web heute sowohl in ökonomischer als auch in sozialer Hinsicht prägen, sind die weithin sichtbaren Folgen dieser großflächigen Landnahme (zum Begriff Dörre 2019).

Vor allem der strukturierende und regulierende Einfluss, den die führenden USamerikanischen Technologiekonzerne Amazon, Apple, Google, Facebook und Microsoft erlangt haben, reicht mittlerweile deutlich über ihre ökonomische Marktmacht hinaus und weit in die Gesellschaft hinein. Diese Konzerne entwickeln und betreiben mit ihren Plattformen die wesentlichen technischen Infrastrukturen und Dienste des Webs, auf die nicht nur private Nutzer, sondern auch viele Unternehmen und öffentliche Einrichtungen heute zurückgreifen (müssen). Als quasihoheitliche Akteure kontrollieren sie die zentralen Zugänge zum Internet, strukturieren und observieren die Bewegungsmöglichkeiten der Nutzer, kuratieren und korrigieren in großem Stil Inhalte, Informationsflüsse und Diskussionen. Als strukturbildende Wirtschaftsakteure arbeiten sie an der möglichst lückenlosen Erfassung, Verarbeitung und Inwertsetzung der Datenspuren, die die Nutzer im Web hinterlassen, und haben damit einen großflächigen Prozess der kommerziellen Vermessung und Kommodifizierung sozialer Aktivitäten und Beziehungen im Onlinebereich in Gang gesetzt, der in der Welt vor dem Internet undenkbar gewesen wäre. Darüber hi- 
naus agieren sie nicht mehr bloß als führende und akzentsetzende Marktteilnehmer, sondern unterhalten und regulieren auch eigene Märkte und Arbeitsbeziehungen, deren Teilnehmerkreis zum Teil weit über ihren Unternehmenszusammenhang hinausreicht (Dolata 2018a, 2019).

Die technische, ökonomische und soziale Regelungshoheit, die vor allem die großen Internetkonzerne - und, in allerdings wesentlich überschaubarerer Weise, auch eine Reihe neuerer und spezialisierterer Internetunternehmen wie Uber, Airbnb, Spotify oder Netflix - mit ihren weitläufig ausgelegten Plattformen erlangt haben, korrespondiert mit einem erheblich schwächeren Einfluss staatlicher oder zivilgesellschaftlicher Akteure auf die Strukturierung und Ausgestaltung des Internets. Nicht nur der Großteil der wirtschaftlichen Aktivitäten, sondern auch weite Teile des privaten Austauschs und der netzbasierten Öffentlichkeit finden heute in privatwirtschaftlich organisierten und gestalteten Räumen und also innerhalb von technischen und sozioökonomischen Ordnungsrahmen statt, die von den entsprechende Angebote bereitstellenden Unternehmen gesetzt werden. Dass die Internetunternehmen mit alldem nicht außerhalb der Gesellschaft stehen, sich regelmäßig mit Interventionen der Politik, den Interessen anderer Wirtschaftsakteure, zivilgesellschaftlichem Protest oder eigenwilligem Nutzerverhalten auseinanderzusetzen haben, steht außer Frage, ändert aber wenig daran, dass sie zu den entscheidenden proaktiven und akzentsetzenden Akteuren der Gestaltung und regulativen Einfassung des Internets geworden sind.

Damit bin ich beim Thema dieses Textes. Wenn das alles so ist, dann stellt sich die Frage, wie, über welche Ansatzpunkte und Mechanismen die Internetunternehmen ihre Rolle als strukturbildende, regelsetzende und handlungskoordinierende Kernakteure des heutigen Internets ausfüllen - und zwar sowohl mit Blick auf genuin soziale als auch auf technische Strukturierungs- und Regulierungsleistungen, die ihre Plattformen auszeichnen. Das betrifft vor allem zwei große Regelungsbereiche, die im Zentrum der folgenden Ausführungen stehen:

- zum einen die eigenständige Organisierung und Regulierung von Märkten für Produkte, Dienstleistungen und Arbeit, auf denen sie als Plattformbetreiber selbst die Marktprozesse koordinieren und die Wettbewerbsbedingungen festlegen, sowie von gesamtwirtschaftlichen Zusammenhängen, wie sie sich etwa in den Plänen andeuten, eigene digitale Währungen einzuführen;

- zum anderen die weitläufige Strukturierung und Kuratierung von Inhalten, Kommunikation und Öffentlichkeiten, durch die die Plattformbetreiber die institutionellen Grundlagen für private Ausdrucks- sowie öffentliche Informations- und Diskursmöglichkeiten legen und damit zum Teil sehr weitreichende soziale Ordnungs- und Regulierungsfunktionen im Web übernehmen.

Beides - Organisierung von Märkten und Gestaltung sozialer Handlungsrahmen - konzentriert sich im heutigen Internet auf wenige privatwirtschaftlich betriebene Plattformen mit großer Reichweite, auf die der weit überwiegende Teil des sozialen und ökonomischen Austauschs entfällt. Beides emergiert nicht einfach aus dem Zusammenspiel einer Vielzahl sozialer Akteure, sondern ist vor allem anderen Ergebnis intentionaler Strukturbildung durch die Plattformbetreiber. Das bezeichne 
ich im Folgenden als Plattform-Regulierung, die in ihrem Kern privatwirtschaftlich organisiert wird und bislang extrem machtasymmetrisch verfasst ist.

Der Beitrag beginnt in Abschnitt 2 mit einer Terrainerkundung, in deren Zentrum privatwirtschaftlich betriebene Internetplattformen als die zentralen soziotechnischen Infrastrukturen des heutigen konsum- und kommunikationsorientierten Webs stehen. Ich diskutiere zunächst relevante Plattformkonzepte, entwickle auf dieser Basis eine eigene Typologie und Arbeitsdefinition der Plattform und skizziere schließlich deren sozioökonomische Grundlagen, die auf den ersten Blick noch nicht sonderlich spektakulär und disruptiv daherkommen.

Das ändert sich mit dem Abschnitt 3, in dem die zwei genannten Regelungsbereiche - Koordinierung von Märkten und Kuratierung von Sozialität - in den Blick genommen werden, die das eigentlich Neue und Disruptive der Internetplattformen ausmachen. Anhand dieser beiden Regelungsbereiche lässt sich die zentrale Bedeutung konkretisieren, die Plattformen als die wesentlichen soziotechnischen Institutionen des heutigen Internets nicht nur für die Organisierung ökonomischer, sondern auch und vor allem für die Prägung und Regelung sozialer Verhältnisse und Prozesse erlangt haben. Dieses Kernstück des Aufsatzes zielt darauf, die empirisch aufspürbaren Strukturierungs- und Organisierungs-, Koordinierungs- und Regelungsformen zu distinkten Mustern und Mechanismen einer soziotechnisch verfassten Regulierung durch Plattformen zu verdichten.

Obgleich die Internetunternehmen zu Kernakteuren einer plattformbasierten Regulierung des Internets geworden sind, bewegen sie sich natürlich nicht außerhalb sozialer Zusammenhänge, gesellschaftlicher Debatten und politischer Eingriffe. Vor dem Hintergrund der kritischer gewordenen öffentlichen Diskussionen zur Macht der Internetkonzerne und ihrer Plattformen werden im Schlussabschnitt Möglichkeiten der Intervention in die Gestaltungshoheit der Plattformbetreiber und Ansatzpunkte einer politischen Einhegung und Regulierung von Plattformen andiskutiert.

\section{Terrainerkundung: Konzeptualisierungen, Varianten und Reichweiten kommerzieller Internetplattformen}

\subsection{Konzeptualisierungen: Fünf Lesarten der Plattform}

Die zahlreichen, zumeist privatwirtschaftlich betriebenen Such-, Networking- und Messaging-, Werbe-, Handels-, Vermittlungs- und Medienangebote im Internet, um die es im Folgenden geht, haben seit den 2000er-Jahren schnell Kontur und raumgreifende Gestalt angenommen und sich seither zu den zentralen Infrastrukturen und Drehscheiben der Informationsbeschaffung, der Kommunikation, der Öffentlichkeit und des Konsums im Netz entwickelt. Zu ihrer Charakterisierung hat sich in der zweiten Hälfte der 2010er-Jahre der Begriff der Plattform eingeschliffen - einer jener zunächst ebenso inklusiven wie unbestimmten ,umbrella terms“, die sich auf sehr verschiedene Weise konkretisieren und kontextualisieren lassen. Fünf für den hier verhandelten Zusammenhang relevante Lesarten werden im Folgenden kurz skizziert und kommentiert. 
Die erste Lesart begreift Plattformen als rechnergestützte, softwarebasierte, programmierbare und algorithmisch strukturierende technologische Architekturen, auf die zahllose spezifische Anwendungen (,applications“) aufsetzen können und die heute die zentralen technischen Infrastrukturen des Netzes bilden (Gillespie 2010, 2014). Sie prägen über ihre technischen Spezifikationen nicht nur die Äußerungsmöglichkeiten individueller Nutzer, sondern strukturieren auch die Handlungsmöglichkeiten von Anbietern etwa kultureller oder politischer Inhalte. Über entsprechende ,software interfaces“ reichen einzelne Plattformen (wie z.B. Facebook) zudem tief ins Netz hinein und ermöglichen so die zentralisierte Abschöpfung und Auswertung zahlloser dezentral entstehender Datenbestände (Gerlitz und Helmond 2013; Helmond 2015). Die vielen sozialen Einschreibungen in diese technischen Infrastrukturen werden zwar bisweilen erwähnt (z. B. bei Kitchin 2014, S. 21 ff.). Wer sie wie konkret sozial konstruiert und implementiert, steht in dieser Lesart der Plattform allerdings nicht im Fokus der Betrachtung.

In der ökonomischen Literatur werden Plattformen vor allem als zwei- oder mehrseitige Märkte begriffen, auf denen die Plattformbetreiber als vermittelnde Intermediäre oder „matchmaker“ fungieren, die mindestens zwei verschiedene Marktakteure - Verkäufer und Käufer, Nutzer und Werbende - zusammenbringen (Rochet und Tirole 2003; Evans und Schmalensee 2016, 2005; Haucap und Stühmeier 2016). Typisch für viele dieser Märkte im Internet sind Netzeffekte mit ihren konzentrationsfördernden Wirkungen. Je stärker eine digitale Plattform genutzt wird und je mehr aktive Mitglieder sie hat, desto interessanter wird sie nicht nur für weitere Nutzer. Die Zahl der regelmäßig aktiven Nutzer auf der einen Seite des Marktes erhöht zugleich die kommerzielle Attraktivität der Plattform etwa für Werbetreibende, Händler oder andere Anbieter auf der anderen Seite des Marktes (Viscusi et al. 2018, S. 383-431). Das Grundprinzip mehrseitiger Märkte ist seit langem bekannt und für viele Wirtschaftszweige bereits seit Jahrzehnten konstitutiv - etwa für Medienangebote wie Buch, Musik, Zeitschriften, Radio und Fernsehen oder für die Reiseund Fahrvermittlung, die nun freilich auf neuer technischer Grundlage zum Teil grundlegend restrukturiert werden. Auch die Vorstellung von einer „gift economy“ (Currah 2007; Elder-Vass 2016), also der kostenfreien Nutzung von Angeboten wie etwa denen von Google oder Facebook, die sich über die andere Seite des Marktes, etwa durch Werbung, finanzieren, hat ihre Vorläufer: Der private Rundfunk und das private Fernsehen beispielsweise funktionieren seit langem nach diesem Prinzip (Evans und Schmalensee 2016, S. 34, S. 197-206).

Aus einer Organisationsperspektive werden kommerzielle Internetplattformen bisweilen als neue idealtypische Form des Unternehmens gefasst, „in which the ,firm' is a set of calls on resources that are then assembled into a performance" (Davis 2016, S. 513). Als Blaupause für derartige ,web page enterprises“ diente in der zweiten Hälfte der 2010er-Jahre oft der Fahrdienstvermittler Uber - ein Unternehmen, das die ökonomische Tragfähigkeit seines Geschäftsmodells seither nicht annähernd nachgewiesen hat - und die stark technisch vermittelte und entgrenzte Art und Weise, wie dort Ressourcen und Arbeitsprozesse organisiert und koordiniert werden: „Hiring, scheduling, performance measuring, and evaluation are now largely in the hands of algorithms“ (Davis 2016, S. 511; auch Rahman und Thelen 2019; Thelen 2018). Diese neuen Ausprägungen in der Organisierung von 
Ressourcen und Arbeit lassen sich als Weiterentwicklungen und Perfektionierungen neoliberaler Markt- und deregulierter Beschäftigungsformen mit neuen technischen Mitteln beschreiben - Trends, die, denkt man etwa an die Ausweitung von Leiharbeitsverhältnissen, bereits seit längerer Zeit zu beobachten sind. Wie raumgreifend sie sind, bleibt in der damit befassten Literatur jedoch regelmäßig unbestimmt. Zumeist wird auf vermeintlich paradigmatische Einzelfälle (,Uberization“) rekurriert, deren Verallgemeinerbarkeit empirisch noch nachzuweisen ist.

In einer grundlegende Veränderungen in der Wirtschaft insgesamt in den Blick nehmenden Perspektive werden Plattformen auch als konstitutiver Ausdruck und Kernelement substanzieller Formveränderungen der kapitalistischen Ökonomie gefasst und mit zum Teil recht weitreichenden Begrifflichkeiten wie ,platform capitalism“ (Srnicek 2017; Langley und Leyshon 2016), „digital platform economy“ (Kenney und Zysman 2016; Zysman und Kenney 2016) oder „Digitaler Kapitalismus“ (Staab 2019) belegt. Betont wird in diesem Zusammenhang nicht nur, dass sich die Plattformökonomie durch starke Konzentrationsprozesse und asymmetrische Machtstrukturen auszeichnet. Ihre Unternehmen agieren in dieser Lesart zugleich als Vorreiter der für die Wirtschaft insgesamt immer wichtiger werdenden Sammlung, Auswertung und Monopolisierung großer Datenbestände, fungieren als organisierende Zentren digitaler ökonomischer Zirkulationsprozesse, koordinieren Arbeitszusammenhänge, Nutzeraktivitäten und die Beiträge externer Produzenten nach ihren Regeln und erweitern die Möglichkeiten der Wertschöpfung um kommodifizierbare Inhalte und Kommunikationen. All diese wichtigen Bausteine fügen sich freilich bislang noch nicht zu einer profunden politischen Ökonomie der Plattform zusammen. Vor allem lassen sie die Frage unbeantwortet, inwieweit sich diese im kommerziellen Internet in der Tat beobachtbaren Mechanismen auf die Gesamtwirtschaft übertragen und zu einem neuen Modell des Kapitalismus oder einer auch klassische Wirtschaftssektoren umfassenden digitalen Ökonomie verallgemeinern lassen.

Eine letzte Lesart, die hier skizziert werden soll, erweitert den Blick auf die soziale, politische und kulturelle Bedeutung von Plattformen (Van Dijck et al. 2018; Van Dijck 2013). Plattformen und die in sie eingeschriebenen sozialen Regeln und Normen, so wird argumentiert, haben soziale Zusammenhänge mittlerweile tief durchdrungen und verändern mit ihren Strukturierungsleistungen die institutionellen Gefüge insgesamt, über die moderne Gesellschaften bis dahin organisiert waren. Das geschieht in dieser Lesart über drei Mechanismen: Über Plattformen werden Daten in großem Stil als Rohstoff abgeschöpft und aufbereitet, Inhalte und Nutzerverhalten sortiert sowie Aktivitäten, Ideen, Emotionen und Objekte zu handelbaren Waren gemacht. Als Plattformgesellschaft wird darauf aufbauend eine Gesellschaft verstanden, in der sowohl ökonomische als auch soziale Prozesse in zunehmendem Maße von global agierenden Plattformunternehmen geprägt sind und in der eine vornehmlich privatwirtschaftlich organisierte Parallelwelt entsteht, die etablierte demokratische Institutionen und Prozesse zunehmend unterläuft (Nieborg und Poell 2018; Zuboff 2019). 


\subsection{Konkretisierungen: Typologie und Arbeitsdefinition der Plattform}

Das Terrain, das in diesen Lesarten aus verschiedenen Blickwinkeln ausgeleuchtet wird, ist freilich recht zerklüftet. Es gibt nicht die kommerzielle Plattform im Internet, sondern verschiedene Varianten, die sich deutlich voneinander unterscheiden und eine typisierende Betrachtung nahelegen. Entlang ihrer Leistungsangebote lassen sich die folgenden Ausprägungen von Plattformen voneinander abgrenzen:

- Suchplattformen, die monopolartig von Google bereitgestellt werden oder sich an Google orientieren;

- Networking- und Messaging-Plattformen, wie sie Facebook (mit WhatsApp und Instagram), Twitter oder Snapchat anbieten;

- Medienplattformen wie zum Beispiel YouTube, Netflix oder Spotify;

- Handelsplattformen wie diejenigen von Amazon, Alibaba, eBay oder Zalando;

- Vermittlungsplattformen zum Beispiel im Bereich von Fahrdienstleistungen (Uber, Lyft), der Reise- und Zimmerbuchung (Airbnb, expedia, booking.com) oder der Partnervermittlung (Match, Parship);

- Cloud-Plattformen wie Amazon Web Services oder Google Cloud Platform, in die individuelle Nutzer, Geschäftskunden und auch staatliche Einrichtungen ihre Daten und deren Verarbeitung auslagern;

- schließlich Crowdsourcing und Crowdfunding-Plattformen wie Amazon Mechanical Turk, das zum IKEA-Konzern gehörende TaskRabbit, Kickstarter oder Indiegogo, die als Drehscheiben für die konkurrenzbasierte Vergabe von Arbeitsaufträgen oder zur Finanzierung von Projekten dienen.

Auf den kleinsten gemeinsamen Nenner gebracht lassen sich all diese Plattformen als digitale, datenbasierte und algorithmisch strukturierende soziotechnische Infrastrukturen charakterisieren, über die Informationen ausgetauscht, Kommunikation strukturiert, Arbeit und Märkte organisiert, ein breites Spektrum an Dienstleistungen angeboten oder digitale und nichtdigitale Produkte vertrieben werden (Kenney und Zysman 2016; Srnicek 2017, S. 43 ff.). Als technische Infrastrukturen basieren sie vor allem auf neuen Möglichkeiten der Erhebung und Verarbeitung großer Datenmengen, der umfassenden digitalen Vernetzbarkeit nicht mehr nur von Medien, Informationen und Kommunikation, sondern auch von materiellen Dingen und Produktionsstrukturen sowie der Sortierung und Koordination dieser Prozesse durch lernende Algorithmen (Gillespie 2014, 2016). Als sozioökonomische Einheiten sind diese Plattformen, obgleich ihr Erfolg (oder Misserfolg) in aller Regel stark von der Zahl sowie den Eigenleistungen, Kommunikationen, Bewertungen und Präferenzen ihrer Nutzer abhängt, nicht crowd- oder sharing-basiert (Sundararajan 2016; Hertwig und Papsdorf 2017), sondern werden durch profitorientierte Unternehmen topdown installiert, organisiert und kontrolliert.

Jenseits dieses kleinsten gemeinsamen Nenners wird das Feld recht heterogen. Nicht nur in Bezug auf klassische ökonomische Kennziffern wie Umsatz, Gewinn oder Beschäftigung (Tab. 1), sondern auch mit Blick auf ihre ökonomische bzw. soziale Reichweite und Bedeutung unterscheiden sich die verschiedenen Internetplattformen deutlich voneinander (Dolata 2018a, 2019; Van Dijck et al. 2018, S. 12-22). 
Tab. 1 Internetunternehmen - ökonomische Eckdaten 2019. (Quellen: Geschäftsberichte der Unternehmen; Presseauswertung. Eigene Zusammenstellung)

\begin{tabular}{|c|c|c|c|c|}
\hline Unternehmen & Umsatz & $\begin{array}{l}\text { Gewinn / } \\
\text { Verlust }\end{array}$ & Kerngeschäft & Beschäftigte \\
\hline $\begin{array}{l}\text { (Ende Ge- } \\
\text { schäftsjahr) }\end{array}$ & $\begin{array}{l}\text { in Mrd. } \\
\text { US-Dollar }\end{array}$ & $\begin{array}{l}\text { in Mrd. } \\
\text { US-Dollar }\end{array}$ & in Prozent vom Umsatz & \\
\hline $\begin{array}{l}\text { Amazon } \\
(12 / 2019)\end{array}$ & 280,52 & $+11,59$ & $\begin{array}{l}\text { Handel und Abonnements } \\
(88 \%) ; \text { Cloud }(12 \%)\end{array}$ & 798.000 \\
\hline $\begin{array}{l}\text { Apple } \\
(9 / 2019)\end{array}$ & 260,17 & $+55,26$ & Geräte ( $82 \%)$; Services $(18 \%)$ & 137.000 \\
\hline $\begin{array}{l}\text { Google } \\
(12 / 2019)\end{array}$ & 161,86 & $+34,34$ & Werbung $(84 \%)$; Cloud $(5,5 \%)$ & 118.899 \\
\hline $\begin{array}{l}\text { Microsoft } \\
(6 / 2019)\end{array}$ & 125,84 & $+39,24$ & $\begin{array}{l}\text { Software und Services }(70 \%) \text {; } \\
\text { Cloud }(30 \%)\end{array}$ & 144.000 \\
\hline $\begin{array}{l}\text { Facebook } \\
(12 / 2019)\end{array}$ & 70,70 & $+18,49$ & Werbung $(98 \%)$ & 44.942 \\
\hline $\begin{array}{l}\text { Netflix } \\
(12 / 2019)\end{array}$ & 20,16 & $+1,87$ & $\begin{array}{l}\text { Filmstreaming / } \\
\text { Abonnement }\end{array}$ & 6.700 \\
\hline $\begin{array}{l}\text { Uber } \\
(12 / 2019)\end{array}$ & 14,15 & $-8,51$ & $\begin{array}{l}\text { Fahrdienstleistungen / } \\
\text { Buchungsgebühren }\end{array}$ & 26.900 \\
\hline $\begin{array}{l}\text { Spotify } \\
(12 / 2019)\end{array}$ & 6,76 & $-0,06$ & $\begin{array}{l}\text { Musikstreaming / } \\
\text { Abonnement und Werbung }\end{array}$ & ca. 4.600 \\
\hline $\begin{array}{l}\text { Airbnb } \\
(12 / 2019)\end{array}$ & 4,80 & $-0,67$ & $\begin{array}{l}\text { Buchung von Unterkünften / } \\
\text { Buchungsgebühren }\end{array}$ & 12.736 \\
\hline $\begin{array}{l}\text { Twitter } \\
(12 / 2019)\end{array}$ & 3,46 & $+1,47$ & $\begin{array}{l}\text { Mikroblogging / } \\
\text { Werbung }\end{array}$ & 4.900 \\
\hline $\begin{array}{l}\text { Snap } \\
(12 / 2019)\end{array}$ & 1,72 & $-1,03$ & $\begin{array}{l}\text { Instant Messaging / } \\
\text { Werbung }\end{array}$ & 3.195 \\
\hline
\end{tabular}

Die führenden Internetkonzerne bieten ein breites Spektrum an aufeinander abgestimmten und vernetzten Diensten und Geschäftsfeldern an, die sie zu weitläufigen, über ihr angestammtes Betätigungsfeld deutlich hinausreichenden soziotechnischen Ökosystemen ausgebaut haben. Google ist längst nicht mehr nur eine Suchmaschine, sondern verfügt mit YouTube auch über den mit Abstand größten Videokanal im Netz, mit Google Play über den neben Apple größten App-Store mit Medieninhalten aller Art, mit Gmail über den führenden E-Mail-Dienst, mit Google Maps über den zentralen Kartendienst, mit Android über das bedeutendste Betriebssystem für mobile Geräte und gehört neben Amazon und Microsoft zu den größten Anbietern von Cloud-Diensten. Facebook beherrscht zusammen mit seinen Töchtern WhatsApp und Instagram unangefochten das Social Networking und Messaging. Auch Apple und Amazon haben sich im vergangenen Jahrzehnt als Komplettanbieter eines breit gefächerten Angebots aus Geräten, Diensten und Medieninhalten profiliert, die sie mittlerweile zum Teil auch selbst produzieren. Die privatwirtschaftliche Regulierung des Internets erfolgt ganz wesentlich über diese weit ausgelegten und tief ins Netz hineinreichenden Plattformen, auf deren Leistungen nicht nur individuelle Nutzer, sondern auch zahlreiche Unternehmen, Medienproduzenten, staatliche Einrichtungen oder andere Plattformunternehmen systematisch zurückgreifen (müssen) (Barwise und Watkins 2018). 
Demgegenüber bieten die zahllosen kleineren Internetunternehmen spezifischere Leistungen auf ihren Plattformen an. In der Regel handelt es sich dabei um singuläre und spezialisierte Konsum- oder Dienstleistungsangebote, die entweder, wie etwa Fahrdienstleistungen, Reisebuchungen, Zimmervermittlungen, Video-on-Demand-Dienste und Shopping-Portale, rein konsumorientiert oder, wie beispielsweise Twitter oder Snapchat, kommunikationsorientiert ausgerichtet sind. Sie bieten ein begrenztes Leistungsspektrum an, das als Geschäft oft traditionellen Wirtschaftssektoren zugeordnet werden kann, die durch die Aktivitäten der neuen Akteure zum Teil radikal neu justiert werden. Uber beispielsweise hat mit neuen Beförderungskonzepten Bewegung in die Märkte für Fahrdienstleistungen und Airbnb neue Dynamik in die Vermittlung von Unterkünften gebracht. Netflix hat sich im vergangenen Jahrzehnt von einem klassischen Videoverleih zum weltweit führenden FilmstreamingDienst mit eigenen Filmproduktionen entwickelt. Viele dieser Plattformen sind auf die infrastrukturellen Angebote der großen Internetkonzerne angewiesen. Netflix oder Spotify beispielsweise laufen komplett über die Server von Amazon Web Services bzw. Google Cloud; zur geografischen Navigation wird nicht nur bei Airbnb der Kartendienst Google Maps genutzt und in das Angebot integriert.

Aus ökonomischer Sicht ist zweierlei bemerkenswert. Zum einen ist das Repertoire an kommerziell tragfähigen Geschäftsmodellen über die Jahre eng begrenzt geblieben. Es konzentriert sich kaum anders als bereits Anfang der 2000er-Jahre, als Plattformen noch unter dem Label „E-Commerce“ diskutiert wurden (Zerdick et al. 2001, S. 167 ff.), auf Werbung, Handel, Subskriptionsmodelle, Vermittlungsgebühren sowie die Aufbereitung von Datenbeständen und den Verkauf von Geräten. Dies trifft nicht nur für kleinere Plattformunternehmen wie Airbnb, Uber, Spotify oder Netflix zu, sondern auch für die führenden Internetkonzerne (Tab. 1).

Bemerkenswert ist zum anderen, dass die gesamtwirtschaftlichen und Beschäftigungseffekte, die die Ausbreitung dieser Plattformen mit sich gebracht hat, bislang sehr überschaubar geblieben sind. Eine empirische Untersuchung des Bureau of Economic Analysis beim U.S. Department of Commerce schätzt für die USA, dass die in der digitalen Wirtschaft insgesamt Beschäftigten, zu der auch die gesamte informations- und kommunikationstechnische Industrie gezählt wird, dort 2016 mit nur 3,9\% zur Gesamtbeschäftigung beigetragen haben. Der Anteil kommerzieller Internetplattformen an der Gesamtbeschäftigung lag bei unter einem Prozent, also noch einmal deutlich niedriger (Barefoot et al. 2018). Und eine Studie des Internationalen Währungsfonds zur Messung der gesamtwirtschaftlichen Effekte der digitalen Ökonomie kommt ebenfalls für die USA zu dem Ergebnis, dass Online-Plattformen und -Services 2015 lediglich 1,5\% zum US-amerikanischen Bruttoinlandsprodukt (BIP) beigetragen haben (International Monetary Fund 2018). Von einer Transformation der Wirtschaft in Richtung eines Plattformkapitalismus oder einer digitalen Plattformökonomie ist dies alles noch weit entfernt.

Die ausgesprochen geringe gesamtwirtschaftliche Bedeutung des hier betrachteten Teilbereichs der (digitalen) Ökonomie, die in den vorgebrachten Zahlen zum Ausdruck kommt, spiegelt allerdings weder den beachtlichen Einfluss der Internetunternehmen auf die Readjustierung ökonomischer Strukturen und Prozesse noch die außerordentliche soziale und gesellschaftspolitische Bedeutung, die sie mittlerweile erlangt haben, adäquat wider. Die schnelle Verbreitung kommerzieller Internet- 
plattformen hat in den vergangenen zwei Jahrzehnten nicht nur massive Umbrüche in einer Reihe von Wirtschaftssektoren, etwa im Handel, auf den Werbemärkten sowie in verschiedenen Medien- und Dienstleistungssektoren, ausgelöst und dort substanzielle Restrukturierungsprozesse in Gang gesetzt. Eine Reihe von Internetunternehmen hat sich zudem als regelsetzende Koordinatoren unternehmenseigener und international ausgerichteter Märkte etabliert. Darüber hinaus werden weite Teile des sozialen Austauschs im Netz - von privater Kommunikation und persönlicher Selbstpräsentation bis hin zu Öffentlichkeiten der unterschiedlichsten Art - mittlerweile von wenigen kommerziell betriebenen Plattformen gebündelt, nach eigenen Maßgaben bewertet und sortiert.

Organisierung von Märkten und Kuratierung von sozialen Zusammenhängen dies sind (neben der Kommodifizierung von Nutzerverhalten; Zuboff 2019; Voß 2020) die wesentlichen Merkmale, die das Neue und Disruptive privatwirtschaftlicher Plattformen ausmachen und sie als zentrale Regulierungsinstanzen im heutigen Internet ausweisen. Sie werden im Folgenden eingehender betrachtet.

\section{Regulierung durch Plattformen: Organisierung von Märkten und Kuratierung von Sozialität}

\subsection{Organisierung von Märkten und gesamtwirtschaftlichen Zusammenhängen}

Auch plattformbetreibende Internetunternehmen agieren natürlich, dies ist zunächst zu betonen, als Akteure auf Märkten und versuchen, mit ihren Expansionsstrategien in neue Marktsegmente einzudringen und diese zu dominieren. Sie stehen dabei in intensivem Wettbewerb sowohl untereinander als auch mit traditionellen Unternehmen aus den Bereichen, die sie herausfordern. Dies betrifft nicht nur kleinere Internetfirmen wie Uber, Airbnb, Spotify oder Netflix, die sich auf den Märkten für Fahrdienstleistungen, für die Vermittlung von Unterkünften oder für Medieninhalte nicht nur mit direkten Konkurrenten auseinandersetzen müssen, sondern sich auch gegenüber den etablierten Anbietern und zum Teil auch gegenüber den führenden Internetkonzernen zu behaupten haben. Auch Letztere bewegen sich keineswegs in wettbewerbsfreien Räumen. Sie dominieren in der einen oder anderen Konstellation zwar wichtige und oft hochkonzentrierte Märkte, allerdings in der Regel nicht als Monopolisten. Dies betrifft die Internetwerbung und die App-Stores ebenso wie Cloud-Dienste, integrierte Medienangebote oder den Handel, die durch duo- oder oligopolistische Strukturen und Konkurrenzmuster geprägt sind. Darüber hinaus ringen die Internetkonzerne regelmäßig auch um die Vorherrschaft bei neuen technischen Trends wie Bild- und Spracherkennung, Machine Learning und virtueller Realität (Dolata 2018a; Parker et al. 2016, S. 210-227). Unübersehbare Konzentrationstendenzen auf internetbasierten Märkten gehen also überall mit scharf geführten Konkurrenzauseinandersetzungen und Strategien der Domänenabsicherung bzw. -erweiterung einher.

Die Internetunternehmen lassen sich allerdings längst nicht mehr nur als mit anderen konkurrierende Marktteilnehmer fassen, sondern agieren deutlich darüber 
hinausgehend auch als Akteure, die eigene Märkte betreiben, koordinieren und kontrollieren. Typisch für diese privatwirtschaftlich verfassten und onlinevermittelten Märkte ist, dass dort die Marktbetreiber die Rolle von Intermediären übernehmen, die die Markthandlungen von Drittanbietern allerdings nicht einfach technisch ermöglichen, sondern sie als regelsetzende Akteure zugleich strukturieren, regulieren und überwachen.

In diese Kategorie fallen einige der großen Plattformen der führenden Internetkonzerne. Amazon unterhält mit Amazon Marketplace die größte Handelsplattform für Drittanbieter im Internet, die mittlerweile einen höheren Umsatz als das eigene Versandgeschäft des Konzerns erzielt. Google betreibt mit YouTube eine zentrale Medienplattform im Web und organisiert über das YouTube-Partnerprogramm die Rahmenbedingungen und Monetarisierungsmöglichkeiten der YouTuber und Influencer sowie professioneller Medienproduzenten. Apple, Google und Amazon verfügen darüber hinaus über große App-Stores, auf denen Softwareentwickler ebenfalls auf der Basis von durch die Marktbetreiber gesetzten Richtlinien und Provisionsmodellen um kommerzialisierbare Aufmerksamkeit konkurrieren (Barwise und Watkins 2018; Khan 2018; Dolata und Schrape 2014). Während die umfangreichen sozialen Regeln und algorithmischen Strukturierungen dieser großen unternehmenseigenen Märkte von den Konzernen als Marktbetreibern weitgehend autonom gesetzt werden können, gilt dies für die neuen onlinevermittelten Märkte für Fahrdienstleistungen und Unterkünfte, die vor allem von Uber und Airbnb repräsentiert werden, in deutlich eingeschränkterem Maße. Auch sie agieren zwar als regelsetzende, koordinierende und sanktionierende Intermediäre, die bestehende (staatliche) Regulierungen systematisch herausfordern, stehen zugleich allerdings unter einem wesentlich stärkeren öffentlichen Legitimations- und politischen Regulierungsdruck (Thelen 2018) - auch, weil auf diesen zwar international angelegten Märkten letztlich Dienstleistungen mit einem starken lokalen bzw. regionalen Bezug angeboten werden: Taxis werden ebenso wie Unterkünfte vor Ort geordert bzw. vermietet.

Die skizzierten unternehmenseigenen Märkte unterscheiden sich von zahlreichen anderen Internetmärkten, auf denen die Unternehmen als mehr oder weniger dominante und akzentsetzende Marktteilnehmer eigene, kommissionierte oder lizenzierte Produkte bzw. Services anbieten - etwa dem Musik- oder Filmstreaming, den CloudServices oder dem Versandhandel. Amazon beispielsweise agiert in seiner Handelssparte in beiden Rollen: Als Online-Händler mit kommissioniertem Angebot ist der Konzern Akteur auf einem Markt, den er dominiert, und mit Amazon Marketplace agiert er zugleich als regelsetzende und koordinierende Instanz eines eigenen, von ihm konstituierten und kontrollierten Marktes. Während kleinere Unternehmen wie Uber oder Airbnb mit den Märkten, die sie organisieren, weitgehend identisch sind, stellen unternehmenseigene Märkte im beschriebenen Sinn für die führenden Internetkonzerne lediglich einen wichtigen Ausschnitt ihrer gesamten Aktivitäten dar.

Die Organisierung und Regulierung dieser unternehmenseigenen Märkte erfolgt über umfangreiche soziotechnische Regelwerke - Markt- und Wettbewerbsregeln, Koordinations-, Kontroll- und Verwertungsmechanismen -, die einerseits in Geschäfts- und Nutzungsbedingungen, Partnerprogrammen oder Entwicklerrichtlinien fixiert sind und andererseits von den Plattformbetreibern in technische Programme und Anweisungen übersetzt werden. Die Plattformbesitzer legen in genuin sozia- 
len Entscheidungen die Ein- und Ausschlusskriterien für Marktteilnehmer fest, formulieren die Marktregeln, Distributions- und Vergütungsstrukturen, entwickeln Produktinformations-, Rating-, Ranking- und Leistungskontrollsysteme, garantieren sichere Bezahlformen und schöpfen die Daten aller Beteiligten lückenlos ab (Kirchner und Beyer 2016; Staab 2019, S. 176ff.). Anders als auf klassischen Märkten konkretisiert sich der dadurch aufgespannte Handlungsrahmen für die Marktteilnehmer und Plattformnutzer allerdings nicht mehr vornehmlich über die soziale Durchsetzung dieser sozialen Regeln, sondern ganz wesentlich über die technischen Infrastrukturen und Programme der Plattform, in die die sozialen Grundlegungen des Marktes - seine strukturellen, regulativen und prozessualen Eigenheiten - als technische Spezifikationen eingeschrieben werden. Die Umsetzung der Marktregeln sowie die konkrete Koordination und Abwicklung aller Marktprozesse erfolgt weitgehend automatisiert und wird algorithmisch gesteuert (Gillespie 2014; Kitchin 2014, S. 15-26, $80 \mathrm{ff}$; Beer 2017).

In die Konstitution dieser privatwirtschaftlich geregelten Märkte sind starke Machtasymmetrien eingelagert - sehr ungleiche soziale Kräfteverhältnisse zwischen den beteiligten Akteuren, die sich auf verschiedenen Ebenen konkretisieren. Erstens verfügen die Plattformbetreiber über eine beträchtliche infrastrukturelle Macht. Sie konzipieren und kontrollieren die technischen Grundlagen, auf deren Basis sich die Marktprozesse entfalten, und fungieren als Gatekeeper, die über den In- bzw. den Ausschluss sowie die Konditionen, denen die Marktteilnehmer unterliegen, entscheiden (Barzilai-Nahon 2008). Damit verbunden zeichnen sich die privatwirtschaftlich organisierten Märkte zweitens durch eine signifikante informationelle Macht der Plattformbetreiber aus, die sich als Hoheit über die anfallenden Daten aller Marktteilnehmer, deren Abschöpfung, Kontrolle und Auswertung darstellt und den Plattformbetreibern einen lückenlosen Überblick über alles, was auf den von ihnen organisierten Märkten geschieht, verschafft. Dabei geht die (vermeintliche) Transparenz der Informations-, Rating- und Rankingsysteme einher mit einer systematischen Opazität ihrer algorithmischen Grundlagen, deren Konzipierung, Veränderung und Weiterverarbeitung für Nutzer, Anbieter, Konsumenten wie auch für staatliche Regulierungsinstanzen eine Blackbox bleiben (Pasquale 2015).

Drittens tragen diese Informationsasymmetrien zur ohnehin marktbeherrschenden Macht der Marktbetreiber bei, die zum Teil im gleichen Segment auch als führende Marktteilnehmer auftreten. Google ist zugleich Medienkonzern mit eigenen kommerziellen Angeboten und Betreiber des Medienkanals YouTube. Apple, Google oder Amazon können über ihre marktförmig organisierten App-Stores zahllose Softwareentwicklungen von Drittanbietern sichten und bei Bedarf davon für ihr eigenes Geschäft profitieren. Und Amazon überblickt auf seinem Marketplace alle Angebote aller Teilnehmer und kann sich so Wettbewerbsvorteile für sein eigenes Handelsgeschäft verschaffen, wie Khan (2018, S. 119) ausführt:

Amazon is exploiting the fact that some of its customers are also its rivals. The source of this power is: (1) its dominance as a platform, which effectively necessitates that independent merchants use its site; (2) its vertical integration - namely, the fact that it both sells goods as a retailer and hosts sales by others as a marketplace; and (3) its ability to amass swaths of data, by virtue of being 
an Internet company. Notably, it is this last factor - its control over data - that heightens the anticompetitive potential of the first two.

Vor allem aber verfügen die Plattformbetreiber viertens über regelsetzende und handlungsstrukturierende Macht und übernehmen quasi-hoheitliche Aufgaben der Marktstrukturierung und -regulierung. Je relevanter eine Plattform für die Visibilität und Abwicklung des Geschäfts wird, desto stärker wirkt der Zwang für die Marktteilnehmer, auf der Plattform präsent zu sein und das eigene Angebot an deren Struktureigenheiten und Regeln anzupassen. Dies betrifft Services wie Reise- und Hotelbuchungen, die mittlerweile kaum noch über die Seiten der direkten Anbieter erfolgen, sondern vornehmlich über Plattformen wie Airbnb, booking.com oder expedia, ebenso wie weite Teile der Kultur- und Medienproduktion - beispielsweise die Angebote klassischer Medienunternehmen, die außerhalb der großen Internetplattformen in der Regel kaum noch wahrgenommen werden. Dadurch verlieren Kultur- und Medienproduzenten nicht nur an Handlungsautonomie und Kontrolle über ihre Distributions- und Kommunikationskanäle. Sie müssen zugleich, wie Nielsen und Ganter (2018, S. 1615) zeigen, die Produktion, Distribution und Verwertung ihrer Inhalte recht weitgehend an den strukturierenden Rahmen und die Regeln der Plattformen anpassen:

Today, they have far less control over the distribution of news than they had in the past. They may reach wider audiences than they can through their own websites and apps, but they do it by publishing to platforms defined by coding technologies, business models, and cultural conventions over which they have little influence and are increasingly dependent.

Mit alldem konstituieren sich privatwirtschaftlich geregelte und soziotechnisch verfasste Marktordnungen im Internet, die sich deutlich von anderen Märkten abheben. Sie sind weder vornehmlich staatlich organisiert, reguliert oder garantiert noch konstituieren sie sich über das selbstorganisierte und deliberative Zusammenspiel verschiedener nicht-staatlicher Akteure (Aspers 2011, S. 148-168; Ahrne et al. 2015), sondern werden von einzelnen Unternehmen installiert, betrieben und kontrolliert. Die Plattformbetreiber agieren dabei nicht als als neutrale Intermediäre, sondern als regel- und akzentsetzende Akteure, die sich selbst mit weitreichenden Befugnissen und Eingriffsmöglichkeiten ausstatten und die wesentlichen Funktionen übernehmen, die die Voraussetzung für die Funktionsfähigkeit und Akzeptanz des Marktes bilden. Und die technischen Infrastrukturen, die die Plattformbetreiber bereitstellen, sind keine neutralen Architekturen, über die lediglich Verbindungen hergestellt werden, sondern bilden durch die in sie eingeschriebenen Regeln die eigentlich handlungsorientierende und prozessstrukturierende institutionelle Grundlage dieser Märkte, an der sich Anbieter, Konsumenten und Nutzer auszurichten haben, wenn sie mitspielen wollen.

Noch einen deutlichen Schritt weiter gehen Pläne, plattformspezifische private Währungen zu etablieren. Zu Ende gedacht könnte damit die beschriebene Privatisierung von Marktordnungen um die wesentlich weiter reichende Perspektive einer privatwirtschaftlichen Regulierung gesamtwirtschaftlicher Zusammenhänge erweitert werden, mit der hoheitliche Aufgaben, die bislang vornehmlich von demokra- 
tisch legitimierten und politisch unabhängigen Einrichtungen wahrgenommen werden, in signifikanter Weise auf private Unternehmen bzw. Unternehmenskonsortien übergingen. Dies beträfe etwa die Geldversorgung und Regulierung der Geldmenge, die Zinspolitik und Sicherung der Preisniveaustabilität oder die Bankenaufsicht, die bislang zu den Domänen der Zentralbanken zählen.

Am fortgeschrittensten sind derartige Pläne bei Facebook. Der Konzern hat Mitte 2019 mit dem Libra-Projekt nicht nur ein erstes Konzept für eine digitale Währung, sondern auch einen dazu passenden Regulierungs- und Institutionalisierungsrahmen vorgestellt (Schmeling 2019; Taskinsoy 2019; Mai 2019). In dessen Zentrum soll die Libra Association stehen - ein Konsortium aus Internetunternehmen, Zahlungsanbietern und weiteren Organisationen -, die als privatwirtschaftliches Pendant und Parallelstruktur zu den Zentralbanken konzipiert ist. Dieses Gremium soll nicht nur für die Konzipierung und Durchsetzung der Libra-Regeln und die technische Infrastruktur der digitalen Währung zuständig sein, sondern exklusiv auch die LibraReserve verwalten, Libra-Geld schöpfen und die Geldmenge steuern, die Zahlungswege kontrollieren und neue Libra-Händler zulassen (Libra Association 2019).

In welchem Umfang diese weitreichenden Pläne Realität werden, ist noch nicht absehbar und muss an dieser Stelle auch nicht diskutiert werden. Wichtig für den hier verhandelten Zusammenhang ist aber ihre allgemeine Stoßrichtung: Sie zielen darauf, die Bedeutung von Zentralbanken und Regierungen in einem zentralen Bereich gesamtwirtschaftlicher Steuerung zu relativieren und durch privatwirtschaftliche Formen gesamtwirtschaftlicher Regulierung zu ergänzen bzw. zu ersetzen - ein Programm der Übernahme quasi-hoheitlicher ökonomischer Regulierungsaufgaben durch die Privatwirtschaft, das nahtlos an die eingangs skizzierten Eckpfeiler der libertären Ideologie anschließt und das, wie wir im Folgenden sehen werden, durch die Übernahme quasi-hoheitlicher sozialer Strukturierungs- und Kuratierungsaufgaben noch einmal wesentlich erweitert wird.

\subsection{Kuratierung sozialer Verhältnisse und Prozesse}

Neben der Organisierung und Regulierung von Märkten haben Plattformen, insbesondere die weitläufig ausgelegten und vernetzten Ökosysteme der führenden Internetkonzerne, wesentliche soziale Ordnungs- und Regulierungsfunktionen im Internet übernommen, die hier zusammenfassend als Kuratierung sozialer Verhältnisse und sozialen Verhaltens bezeichnet werden (Abb. 1). Über ihre zahlreichen Dienste und Angebote filtern sie Informations- und Kommunikationsprozesse, prägen individuelles Verhalten wie organisationales Handeln und strukturieren soziale Beziehungen und Öffentlichkeiten der unterschiedlichsten Art - und das in weit umfassenderer Weise, als dies selbst großen Medienkonzernen jemals möglich war (Couldry und Hepp 2016, S. 34-56; Lobigs und Neuberger 2018). Während Medienkonzerne als mächtige meinungsbildende Akteure mit allerdings begrenzter Reichweite in die Gesellschaft und ihr Institutionengefüge eingebettet blieben, konstituieren die großen Plattformen mit ihren eigenen Regelwerken, Strukturierungs-, Selektions-, Überwachungs- und Sanktionierungsleistungen nicht weniger als die institutionellen Grundlagen einer privatwirtschaftlich verfassten Gesellschaftlichkeit im Internet, die 


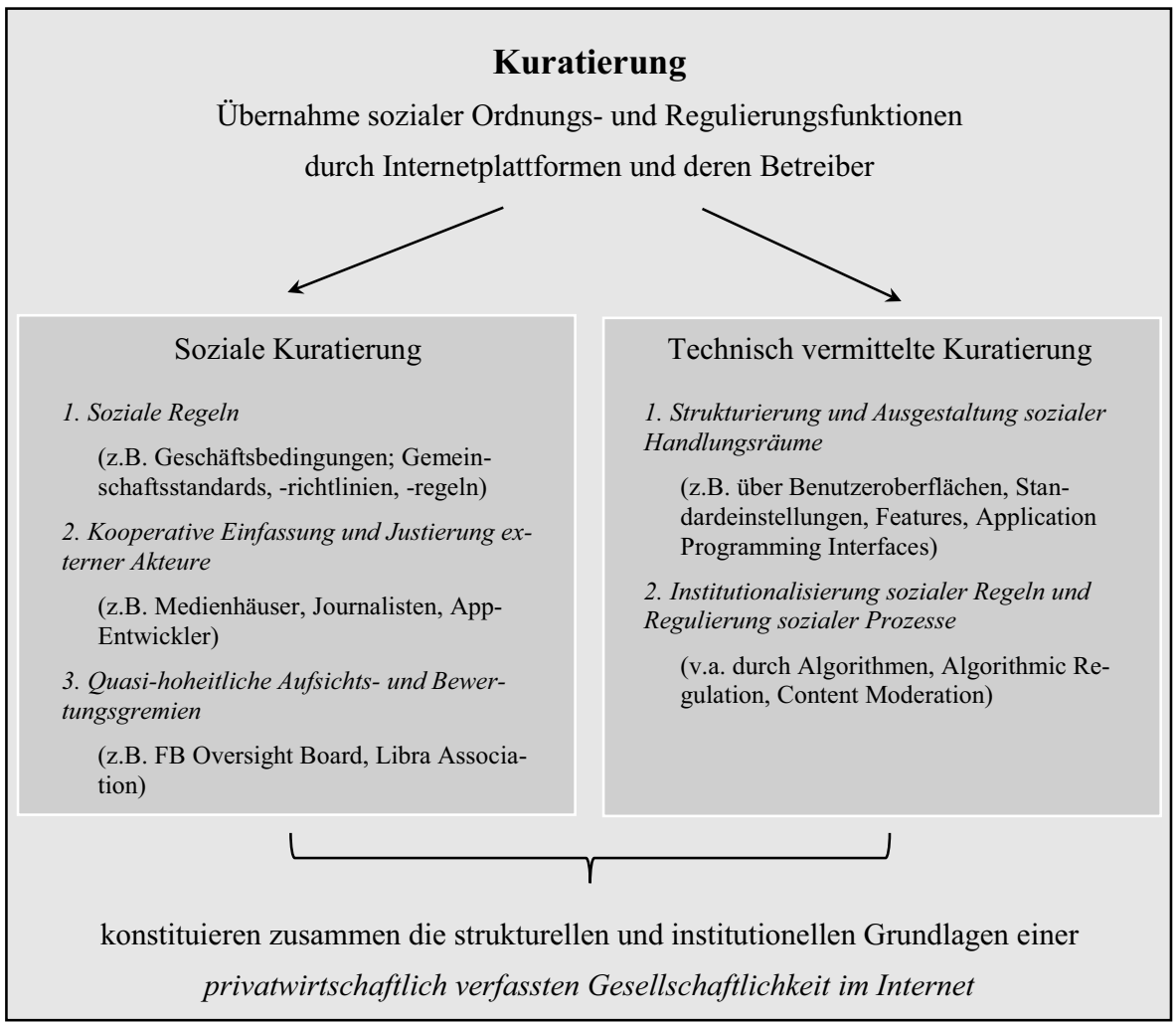

Abb. 1 Soziale und technisch vermittelte Kuratierung: Ebenen und Ansatzpunkte. (Quelle: eigene Überlegungen)

sich in den vergangenen zwei Jahrzehnten weitgehend entkoppelt von den demokratischen Institutionen und staatlicher Einflussnahme herausgebildet haben.

Die Basis der Kuratierung bilden wiederum verpflichtende und sanktionierbare soziale Regeln. Sie finden ihren Ausdruck in den Geschäftsbedingungen, vor allem aber in Gemeinschaftsstandards (Facebook), -richtlinien und -regeln (YouTube; Twitter), in denen die Plattformbetreiber detailliert ausformulieren, was nach ihrer Maßgabe als politisch inakzeptabel, gewaltverherrlichend, terroristisch, anstößig, obszön, erotisch oder pornografisch gilt und entsprechend sanktioniert wird. In die Weiterentwicklung dieser Richtlinien, die den rechtlichen und normativen Rahmen für alle sozialen Aktivitäten auf den Plattformen aufspannen, fließen zwar auch gesellschaftliche Meinungslagen und politische Interventionen ein, auf die die Internetunternehmen zu reagieren haben. Letztere verlieren damit bislang allerdings nicht die Hoheit über die Regelsetzung und -durchsetzung auf ihren Plattformen, über die sie in letzter Instanz allein entscheiden.

Diese Formen sozialer Kuratierung werden durch verschiedene Ansatzpunkte einer technisch vermittelten Kuratierung nicht bloß in Technik übersetzt, sondern noch einmal signifikant erweitert. Aus der techniksoziologischen Forschung ist seit langem bekannt, dass in Technik immer auch soziale Regeln, Normen, Anleitungen und 
Kontrollmechanismen durch die Hersteller eingebaut sind, die auf die Aktivitäten ihrer Nutzer wie Institutionen wirken und deren Handeln mitprägen (Dolata 2013, S. 32 ff.). In den 1990er-Jahren hatte Christiane Floyd (1992) Softwareentwicklung als Realitätskonstruktion charakterisiert und der bereits erwähnte Lawrence Lessig (1999) ebenfalls mit Blick auf Software die Metapher „,code is law“ formuliert und damit all die Anweisungen und Prozeduren, die in Software eingeschrieben werden, in ihrer handlungsregelnden Bedeutung mit dem Recht und anderen sozialen Regelsystemen gleichgesetzt. Bereits zwei Jahrzehnte zuvor hatte Langdon Winner (1980, S. 127f.) technische Arrangements als strukturbildende und regelsetzende Ordnungsmuster charakterisiert:

The things we call "technologies" are ways of building order in our world. [...] In that sense technological innovations are similar to legislative acts or political foundings that establish a framework for public order.

Diese klassische Sicht auf die strukturbildenden und institutionellen Effekte von Technik lässt sich im Plattformkontext zum einen als technisch vermittelte Strukturierung und Ausgestaltung von sozialen Handlungsräumen konkretisieren, die die Aktivitäten von Nutzern der unterschiedlichsten Art sowohl ermöglichen als auch kanalisieren. Hierzu zählen die vorgegebenen Benutzeroberflächen und Standardeinstellungen der Plattformen, die eine handlungsstrukturierende Wirkung haben, indem sie bestimmte Aktivitäten ermöglichen und andere ausschließen. Auch die zahlreichen in die Plattformen eingebetteten Features (wie die Reactions bei Facebook oder der Trending Button bei Twitter) lassen sich als in Technik eingeschriebene handlungsorientierende und meinungsbildende Strukturelemente fassen. Über Application Programming Interfaces (APIs) sind zudem die Webauftritte zahlloser dritter Akteure in den Handlungsraum der Plattformen integriert und weitreichende Verknüpfungen der Plattformen mit externen Websites, Plattformen und Apps eingerichtet worden. Das hat zu einer signifikanten Entgrenzung der technischen Architekturen und damit auch der sozialen Bedeutung und Reichweite von Plattformen im Internet geführt, die in der Literatur als ,platformization“ beschrieben worden ist: Einerseits reicht der strukturierende Einfluss einzelner Plattformen dadurch mittlerweile deutlich über ihre ursprüngliche Domäne hinaus und tief in das Social Web hinein und prägt den Handlungsrahmen zahlloser anderer Netzakteure. Und andererseits ermöglicht die Integration von Dritten es den Plattformbetreibern, auch die dort anfallenden plattformexternen Datenbestände systematisch abzuschöpfen und für ihre eigene Datensammlung bzw. -auswertung zu nutzen (Nieborg und Helmond 2019; Helmond 2015; Gerlitz und Helmond 2013).

Diese strukturbildenden Effekte von Technik werden zum anderen ergänzt durch Ansatzpunkte einer technisch vermittelten Institutionalisierung sozialer Regeln und Regulierung sozialer Prozesse, die vor allem durch den Einsatz von Algorithmen umgesetzt und in der Literatur als ,algorithmic governance“, ,algorithmic regulation“ oder ,algorithmic content moderation“ bezeichnet wird (Gillespie 2014, 2016; Kitchin 2014; Just und Latzer 2017; Beer 2017; Yeung 2018; Katzenbach und Ulbricht 2019; Gorwa et al. 2020). Algorithmen setzen die sozialen Regeln und Normen, die auf den Plattformen gelten, als technische Anweisungen um, überwachen und sanktionieren die Aktivitäten der Teilnehmer, entscheiden entlang von in sie eingeschrie- 
benen sozialen Relevanzkriterien darüber, was wichtig ist und was nicht, selektieren, aggregieren und ranken auf dieser Grundlage Informationen, Nachrichten, Videos oder Fotos, strukturieren private Informations- und Kommunikationsprozesse sowie öffentliche Diskursmöglichkeiten, tragen zur Konstituierung von Öffentlichkeiten und Gemeinschaften bei, die es ohne sie gar nicht oder so nicht geben würde - und werden damit zum Nukleus einer technisch vermittelten Rahmung, Kontrolle und Kuratierung sozialen Handelns.

Die regulative Eingriffstiefe von Algorithmen erhöht sich noch einmal dadurch, dass sie schnell und radikal verändert werden können. Entsprechende Neujustierungen werden etwa beim PageRank-Algorithmus der Google-Suche, beim YouTubeAlgorithmus oder beim Newsfeed-Algorithmus von Facebook regelmäßig durch die Plattformbetreiber vorgenommen und rekonfigurieren die auf den Plattformen präsentierte Idee von sozialer Wirklichkeit zum Teil signifikant. Änderungen des Newsfeed-Algorithmus beispielsweise verändern nicht nur unmittelbar das, was die Nutzer an persönlichen Posts und Nachrichten zu sehen bekommen, sondern haben auch massive Auswirkungen auf die Wahrnehmung und den ,web traffic“ öffentlichrechtlicher Medienanstalten oder privater Medienhäuser, deren Performanz mittlerweile hochgradig abhängig von ihrer Präsenz auf den Plattformen ist (Nielsen und Ganter 2018; Van Dijck et al. 2018, S. 49-72). Algorithmen, die die Grundlage jeglicher Suche und Information, Kommunikation und Interaktion auf diesen Plattformen bilden, sind mit alldem hochpolitische Programme, die distinkte, selektive und zunehmend personalisierte soziale Wirklichkeitsangebote auf der Grundlage von Kriterien konstruieren, die sowohl für die Einzelnen als auch für Organisationen und die Politik völlig undurchsichtig bleiben.

Nun wirken, allgemein gesprochen, in Technik eingeschriebene soziale Strukturierungen und Regeln mit ihren institutionellen und regulierenden Eigenheiten natürlich nie handlungsdeterminierend, sondern sind, ähnlich wie Gesetze, Vorschriften, soziale Verhaltensnormen oder Werte auch, interpretierbar - und werden nicht nur durch die Hersteller und Betreiber der Technik, sondern etwa im Ergebnis politischer Interventionen, gesellschaftlicher Auseinandersetzungen oder eigenwilligen Nutzerverhaltens immer wieder angepasst, modifiziert oder auch wieder außer Kraft gesetzt. Dies trifft, konkreter gesprochen, auch für Algorithmen zu:

Algorithms are not just what their designers make of them, or what they make of the information they process. They are also what we make of them day in and day out - but with this caveat: because the logic, maintenance, and redesign of these algorithms remain in the hands of the information providers, they are in a distinctly privileged position to rewrite our understanding of them. (Gillespie 2014, S. 187)

Der von Gillespie eingeschobene Vorbehalt ist wichtig und markiert einen wesentlichen und generalisierbaren Unterschied zwischen Technik als Institution und sozialen Institutionen. Während die sozialen Institutionen demokratischer Gesellschaften in aller Regel in öffentlichen Diskursen und politischen Aushandlungen Gestalt annehmen und der demokratischen Legitimation bedürfen, sind institutionelle Einschreibungen in Technik zumeist die Domäne ihrer (privatwirtschaftlichen) Hersteller und kaum ex ante öffentlich verhandel- oder gestaltbar. 
Die hier beschriebenen zwei zentralen Ebenen sozialer und technisch vermittelter Kuratierung werden angereichert durch zwei weitere Ausprägungen sozialer Kuratierung. Zum einen wird die algorithmische Strukturierung und Sortierung von Medieninhalten und -öffentlichkeiten seit Mitte der 2010er-Jahre ergänzt um Initiativen der Plattformbetreiber, die auf eine stärkere kooperative Einfassung von Medienhäusern und Journalisten zielen (Bell 2018). Dazu zählen Projekte wie die Google News Initiative (https://newsinitiative.withgoogle.com/) oder das Facebook Journalism Project (https://www.facebook.com/journalismproject), die darauf ausgelegt sind, Medienkonzerne und -anstalten, Redaktionen und mediennahe Organisationen über von den Internetkonzernen organisierte Meetings und Schulungskurse, die Entwicklung von Programmen zum Ausbau digitaler Nachrichtendienste sowie die Vergabe von Fördermitteln enger an ihre Plattformen zu binden und stärker an deren Operations- und Verwertungslogiken auszurichten.

Einen wesentlichen Schritt weiter geht zum anderen der Aufbau eines konzerneigenen Aufsichtsgremiums bei Facebook, das sich mit der Überwachung, Moderation und Bewertung von Inhalten auf der Plattform befasst. Das mit externen Experten besetzte und vom Konzern finanzierte Oversight Board, das 2020 seine Arbeit aufnimmt, soll nicht nur die Umsetzung der in den Community Standards fixierten sozialen Regeln überwachen und weiterentwickeln, sondern hat auch die Befugnis, über strittige Inhalte zu richten und sie gegebenenfalls von der Plattform entfernen zu lassen (Harris 2020). Neben der Libra Association verfügt der Konzern damit über ein zweites Gremium mit quasi-hoheitlicher Funktion, das als eine Art Verfassungsgericht und Kontrollausschuss angelegt ist, ohne allerdings über eine vergleichbare demokratische Legitimation zu verfügen und Einfluss auf grundlegende Unternehmensentscheidungen ausüben zu können. Während das Libra-Projekt von Facebook proaktiv vorangetrieben wird, ist das Oversight Board erkennbar als domänenabsichernde Reaktion auf kritischer werdende politische Diskussionen um eine stärkere Regulierung von Internetplattformen auf den Weg gebracht worden. Im Kern zielen beide Projekte darauf, im Kontext der Plattform weitläufige quasi-hoheitliche Parallelstrukturen zu den demokratisch legitimierten gesellschaftlichen Institutionen aufzubauen.

All das zusammengenommen sind die führenden Internetkonzerne, die dies vor allem betrifft, mittlerweile weit mehr als Infrastrukturanbieter, die Konnektivität ermöglichen, Medienkonzerne, die über ein breites Portfolio an eigenen Medienangeboten verfügen, oder Werbe-, Handels-, Hardware- und Dienstleistungsunternehmen, die mit ihren klassischen Geschäften nach wie vor den Großteil ihrer Umsätze und Gewinne erzielen. Die wenigen großen Plattformen, die heute weite Teile des privaten und öffentlichen Lebens im Internet sowohl ermöglichen als auch prägen, lassen sich deutlich darüber hinausgehend als ausdifferenzierte gesellschaftliche Strukturen mit distinkter institutioneller Grundlage fassen, die die Plattformbetreiber über eigene Regeln, Regulierungen und Gremien maßgeblich prägen und kontrollieren. Dies reicht bis hin zur Übernahme bislang staatlichen Instanzen vorbehaltener, quasi-hoheitlicher Aufgaben durch die Unternehmen, die sich demokratischer Legitimation und Kontrolle weitgehend entziehen können. 


\section{Ausblick: Regulierung von Plattformen? Ansatzpunkte und Grenzen politischer Intervention}

Die ökonomische, vor allem aber die soziale Gestaltungsmacht, die die führenden Internetkonzerne mit ihren Plattformen erlangt haben, wird durch nicht-hierarchische Vorstellungen von einer Internet-Governance, die auf „low formalization, heterogeneous organizational forms, large numbers of actors and massively distributed authority and decision-making power" abhebt (Van Eeten und Mueller 2012, S. 730), eher camoufliert als offengelegt. Das, was ich im vorangegangenen Kapitel ausgeführt habe, bezeichne ich demgegenüber als Regulierung durch Plattformen: als intentionale Strukturierung und federführende Gestaltung der Rahmenbedingungen nicht nur für ökonomische Marktbeziehungen und Prozesse, sondern in deutlich umfassenderer Weise auch für größere soziale Zusammenhänge, die durch die Internetunternehmen als Plattformbetreiber vorgenommen und entlang ihrer ökonomischen Verwertungsinteressen ausgerichtet werden.

Das heißt natürlich weder, dass diese Regulierungsaktivitäten die Handlungen anderer Akteure determinieren würden, noch, dass die Internetunternehmen mit ihren Plattformen außerhalb der Gesellschaft stünden und entkoppelt von kollektivem Nutzerverhalten, öffentlichen Diskursen und Meinungslagen, politischen Interventionen oder den Interessen anderer Wirtschaftsakteure agieren könnten. Gestaltungsmacht mag wie im hier vorliegenden Fall sehr asymmetrisch verteilt sein, ist aber nie absolut - etwas, über das die Einen verfügen und die Anderen nicht -, sondern immer Ausdruck komplexer, oft umkämpfter und nicht selten volatiler sozialer Kräfteverhältnisse, aus denen die Einen mehr herausholen können als die Anderen (Dolata und Schrape 2018). Diese Anderen und ihre Interventionsmöglichkeiten stehen im Zentrum der abschließenden Ausführungen. Mit Fokus auf die besonders einflussreichen Plattformen werden im Folgenden zwei Ebenen sozialer bzw. politischer Einflussnahme voneinander unterschieden und vier Möglichkeiten politischer Intervention ausgelotet.

Die erste Ebene lässt sich als zivilgesellschaftliche Intervention bezeichnen. Die Internetkonzerne haben in schneller Folge nicht nur auf Veränderungen der sehr dynamischen technologischen und ökonomischen Umgebungen, in denen sie sich bewegen, sondern auch auf gesellschaftlichen oder politischen Druck zu reagieren, der seit der zweiten Hälfte der 2010er-Jahre signifikant zugenommen hat. Ihre großen Plattformen sind nicht nur existenziell auf die Beiträge, Aktivitätsdichte und Akzeptanz ihrer Nutzer angewiesen, die sich die Angebote der Plattformen zum Teil recht eigenwillig aneignen, sie umnutzen oder auch ablehnen und von den Plattformbetreibern entsprechend sensibel behandelt werden müssen. Die Internetkonzerne stehen seither auch unter intensiver Beobachtung einer aufmerksamer gewordenen medialen und politischen Öffentlichkeit. Investigative Journalisten, netzpolitische Blogs und die klassischen Medien befassen sich mittlerweile ausführlich mit den verschiedenen Facetten ihrer sozialen und ökonomischen Macht: etwa mit intransparenten Geschäftspraktiken und marktbeherrschenden Stellungen, mit umstrittenen sozialen Richtlinien und opaken Algorithmen, mit wiederholten Verletzungen der Privatsphäre und der Überwachung der Nutzer, mit Datenskandalen (wie den um Cambridge 
Analytica), der Verbreitung von Fake News oder der Nutzung von Plattformen zur Beeinflussung von Wahlen (wie der US-amerikanischen Präsidentenwahl 2016).

Insbesondere die Medien, aber auch andere zivilgesellschaftliche Akteure haben damit in den vergangenen Jahren zu einer deutlich kritischeren Bewertung der Plattformen sowohl im öffentlichen Diskurs als auch in der Politik beigetragen, die von deren Betreibern nicht einfach ignoriert werden kann - vor allem dann nicht, wenn sich die Diskussionen zu ernstzunehmenden Forderungen nach einer stärkeren öffentlichen Kontrolle und staatlichen Regulierung der Plattformen verdichten. Die Internetkonzerne, vor allem Facebook und Google, haben darauf mit einer Reihe von Transparenzinitiativen sowie Versuchen einer engeren und institutionalisierten Einbindung zivilgesellschaftlicher Akteure in ihre eigenen Regulierungsstrukturen reagiert (etwa durch die Einrichtung des Oversight Board bei Facebook) (Gorwa 2019).

Die Effekte, die zivilgesellschaftliche Interventionen erzielen können, sind nicht zu unterschätzen: Sie können, wenn sie mit entsprechendem Druck vorgebracht werden und auf große gesellschaftliche Akzeptanz stoßen, schnelle und zum Teil auch substanzielle Anpassungsreaktionen bei den Internetkonzernen auslösen - allerdings ohne dass dadurch deren Ordnungsfunktionen und Regelungshoheit infrage stünden. Auf zivilgesellschaftlichen Druck können die Konzerne im Modus der Freiwilligkeit reagieren, entlang ihrer selbst gesetzten Maßstäbe und dann, wenn es ihnen probat erscheint. Das bleibt unverbindlich und hat mit einer Regulierung von Plattformen nichts zu tun, die demgegenüber ganz wesentlich in der Durchsetzung demokratisch entwickelter und rechtlich verbindlicher öffentlicher Regeln besteht, die von den Plattformbetreibern einzuhalten sind.

Parallel zur Zunahme zivilgesellschaftlicher Interventionen haben sich - dies ist die zweite Ebene externer Einflussnahme - seit der zweiten Hälfte der 2010erJahre auch die staatlichen Bemühungen um eine solche politische Regulierung und Kontrolle der großen Plattformen intensiviert. In Europa lassen sich entsprechende Aktivitäten bislang vor allem auf zwei Handlungsfeldern verorten:

- als Versuche der Eingrenzung von ökonomischer Marktmacht, vorgebracht vor allem von der Europäischen Kommission, die seither eine Reihe von Verstößen der Internetkonzerne gegen das EU-Kartellrecht verfolgt und wiederholt vor allem gegen Google und Facebook hohe Geldbußen verhängt hat - z.B. wegen Missbrauchs ihrer marktbeherrschenden Stellung in der Online-Werbung, bei Suchmaschinen oder beim mobilen Betriebssystem Android (Viscusi et al. 2018, S. 404-419; Haucap und Stühmeier 2016; European Commission 2019);

- als Bemühungen um rechtlich-regulative Eingriffe in die soziale Regelungshoheit der Plattformen - etwa in Gestalt der europäischen Datenschutz-Grundverordnung (DSGVO), des vom Europäischen Gerichtshof mit einem Grundsatzurteil eingeführten „Rechts auf Vergessen“ im Internet oder des deutschen NetzwerkDurchsetzungsgesetzes (NetzDG), das die Anbieter führender sozialer Netzwerke wie Facebook, YouTube oder Twitter dazu verpflichtet, rechtswidrige Inhalte zeitnah zu sperren bzw. von ihren Plattformen zu entfernen und turnusmäßig darüber zu berichten (Schulz 2018; Chenou und Radu 2019). 
Die Reichweite dieser politischen Interventionen ist bislang ausgesprochen begrenzt geblieben. Vor allem die bisherigen staatlichen Versuche eines Eingriffs in die soziale Regelungshoheit der Plattformbetreiber haben paradoxerweise die Regulierungsmacht der Plattformen eher gestärkt, indem sie hoheitliche Kernfunktionen der Rechtsprechung und -durchsetzung auf privatwirtschaftliche Akteure übertragen und diese Verlagerung mit politischer Legitimation versehen haben. Das NetzwerkDurchsetzungsgesetz beispielsweise hat wenig daran geändert, dass Unternehmen wie Facebook, Google oder Twitter weitgehend selbst darüber entscheiden, welche Inhalte sie löschen und welche nicht, hat aber zugleich die Rolle der Unternehmen als Content-Moderatoren und entscheidende Bewertungs- bzw. Selektionsinstanzen von Inhalten gestärkt. Auch die Umsetzung des Rechts auf Vergessen ist den Plattformen selbst vorbehalten geblieben, die damit in das Rechtssystem integriert und auch von der Politik als privatwirtschaftliche Instanzen mit quasi-hoheitlichen Aufgaben aufgewertet worden sind. Chenou und Radu (2019, S. 74 und 96f.) haben das treffsicher als ,outsourcing of important governance practices to private intermediaries" beschrieben und auf die systematische Abhängigkeit der staatlichen Regulierung von der Kooperationsbereitschaft der Plattformbetreiber hingewiesen:

In creating new rights, public actors foster strong regulations they may not be able to implement themselves without the collaboration of private actors. More than a transformation of the state, the resulting hybridization of governance also entails a transformation of private actors. In the process, some private actors are given new responsibilities in the governance of technologies and technologyenabled markets. As the case of the "right to be forgotten" showed, Google becomes inserted in the European legal system as a first instance to look at cases of online privacy protection triggered by individual requests.

Insgesamt sind die bislang verfolgten politischen Regulierungsansätze nicht dazu geeignet, substanziell korrigierend oder kontrollierend in die Regelungshoheit der Plattformbetreiber einzugreifen. Weiter reichende Vorschläge, deren Umsetzung der staatlichen Seite etwas mehr Gewicht in dieser sehr ungleichen privat-staatlichen Regulierungskonstellation verleihen könnten, sind zwar in der Diskussion, werden aber politisch bislang nicht konkretisiert. Dazu zählen

- über Forderungen nach einer rigideren Kontrolle marktbeherrschender Stellungen hinausgehende Überlegungen in Richtung einer radikalen Entflechtung der weitläufig miteinander vernetzten Plattformen der Internetkonzerne (Reich 2018) etwa der Ausgliederung von YouTube und anderer Plattformen aus dem GoogleKonzern oder der Aufspaltung des Ökosystems von Facebook, Instagram und WhatsApp -, die allerdings weniger mit einer Begrenzung ökonomischer Marktmacht begründet als mit dem Ziel verfolgt werden sollte, die außerordentliche soziopolitische Gestaltungsmacht der Internetkonzerne durch das zugegeben brachiale Mittel der Zerschlagung einzugrenzen;

- die Idee der Einrichtung öffentlicher Aufsichts- und Regulierungsagenturen etwa auf europäischer und US-amerikanischer Ebene, die, parlamentarisch kontrolliert sowie mit anerkannten und öffentlich bestellten Experten besetzt, als demokratisch legitimierte Alternativen zu konzerneigenen Aufsichtsgremien wie dem 
Oversight Board von Facebook angelegt und mit weitreichenden Informations-, Kontroll- und Sanktionsrechten gegenüber den Plattformen ausgestattet werden müssten. Algorithmische Filterfunktionen, Ranking- und Ratingprinzipien könnten dort ebenso offengelegt, kontrolliert und mit Auflagen versehen werden wie Community Standards und darauf aufbauende Such- und Selektionskriterien (Dolata 2018b; Kommission Wettbewerb 4.0 2019, S. 80 ff.).

Auch der Vorschlag öffentlicher Aufsichts- und Regulierungsagenturen liefe, wenn er umgesetzt würde, selbst im besten Fall allerdings nicht auf eine gleichberechtigte privat-staatliche Ko-Regulierung von Plattformen hinaus - schon aufgrund der extremen Informations- und Wissensasymmetrien zwischen den Beteiligten: Politische Regulierungsinstanzen wissen grundsätzlich weit weniger über die weitläufigen soziotechnischen Systeme und Systemzusammenhänge, die sie regulativ einhegen sollen, als diejenigen, die diese Systeme entwickelt haben und betreiben. Die Gestaltungs- und Regelungshoheit verbliebe auch in diesem Fall in erster Linie bei den Plattformbetreibern, deren Aktivitäten dann aber immerhin von einer demokratisch legitimierten Instanz regelmäßig evaluiert, kontrolliert und sanktioniert werden könnten.

Funding Open Access funding provided by Projekt DEAL.

Open Access Dieser Artikel wird unter der Creative Commons Namensnennung 4.0 International Lizenz veröffentlicht, welche die Nutzung, Vervielfältigung, Bearbeitung, Verbreitung und Wiedergabe in jeglichem Medium und Format erlaubt, sofern Sie den/die ursprünglichen Autor(en) und die Quelle ordnungsgemäß nennen, einen Link zur Creative Commons Lizenz beifügen und angeben, ob Änderungen vorgenommen wurden.

Die in diesem Artikel enthaltenen Bilder und sonstiges Drittmaterial unterliegen ebenfalls der genannten Creative Commons Lizenz, sofern sich aus der Abbildungslegende nichts anderes ergibt. Sofern das betreffende Material nicht unter der genannten Creative Commons Lizenz steht und die betreffende Handlung nicht nach gesetzlichen Vorschriften erlaubt ist, ist für die oben aufgeführten Weiterverwendungen des Materials die Einwilligung des jeweiligen Rechteinhabers einzuholen.

Weitere Details zur Lizenz entnehmen Sie bitte der Lizenzinformation auf http://creativecommons.org/ licenses/by/4.0/deed.de.

\section{Literatur}

Abbate, J. (1999). Inventing the Internet. Cambridge: MIT Press.

Ahrne, G., Aspers, P., \& Brunsson, N. (2015). The organization of markets. Organization Studies, 36(1), $7-27$.

Aspers, P. (2011). Markets. Cambridge: Polity Press.

Barbrook, R., \& Cameron, A. (1996). The Californian ideology. Science as Culture, 6(1), 44-72.

Barefoot, K. et al. (2018). Defining and measuring the digital economy. Working Paper. Washington D.C.: Bureau of Economic Analysis.

Barlow, J. P. (1996). A declaration of the independence of cyberspace. Electronic Frontier Foundation (8. Feb. 1996). https://www.eff.org/cyberspace-independence. Zugegriffen: März 2020.

Barwise, P., \& Watkins, L. (2018). The evolution of digital dominance: How and why we got to GAFA. In M. Moore \& D. Tambini (Hrsg.), Digital dominance. The power of Google, Amazon, Facebook, and Apple (S. 21-49). Oxford: Oxford University Press. 
Barzilai-Nahon, K. (2008). Toward a theory of network gatekeeping: A framework for exploring information control. Journal of the American Society for Information Science and Technology, 59(9), 1493-1512.

Beer, D. (2017). The social power of algorithms. Information, Communication \& Society, 20(1), 1-13.

Bell, E. (2018). The dependent press. How Silicon Valley threatens independent journalism. In M. Moore \& M. Tambini (Hrsg.), Digital dominance. The power of Google, Amazon, Facebook, and Apple (S. 241-261). Oxford: Oxford University Press.

Chenou, J.-M., \& Radu, R. (2019). The ,right to be forgotten“: Negotiating public and private ordering in the European Union. Business \& Society, 58(1), 74-102.

Couldry, N., \& Hepp, A. (2016). The mediated construction of reality. Cambridge: Polity.

Currah, A. (2007). Managing creativity: The tensions between commodities and gifts in a digital networked environment. Economy and Society, 36(3), 467-494.

Davis, G. F. (2016). What might replace the modern corporation? Uberization and the web page enterprise. Seattle University Law Review, 39, 501-515.

Van Dijck, J. (2013). The culture of connectivity. A critical history of social media. Oxford: Oxford University Press.

Van Dijck, J., Poell, T., \& De Waal, M. (2018). The platform society. Public values in a connective world. Oxford: Oxford University Press.

Dolata, U. (2013). The transformative capacity of new technologies. A theory of sociotechnical change. London: Routledge.

Dolata, U. (2018a). Internet companies: Market concentration, competition and power. In U. Dolata \& J.F. Schrape, Collectivity and power on the Internet. A sociological perspective (S. 85-109). Cham: Springer.

Dolata, U. (2018b). Big Four - Die digitale Allmacht? Blätter für deutsche und internationale Politik, $63(5), 81-86$.

Dolata, U. (2019). Privatization, curation, commodification. Commercial platforms on the Internet. In C. Musik \& A. Bogner (Hrsg.), Digitalization and society. A sociology of technology perspective on current trends in data, digital security and the Internet. Supplement zu Bd. 44 der Österreichischen Zeitschrift für Soziologie (S. 181-197). Wiesbaden: Springer VS.

Dolata, U., \& Schrape, J.-F. (2014). App-Economy: Demokratisierung des Software-Marktes? Technikfolgenabschätzung - Theorie und Praxis, 23(2), 76-80.

Dolata, U., \& Schrape, J.-F. (2018). Collectivity and power on the Internet. A sociological perspective. Cham: Springer.

Dörre, K. (2019). Risiko Kapitalismus. Landnahme, Zangenkrise, Nachhaltigkeitsrevolution. In K. Dörre, H. Rosa, K. Becker, S. Bose \& B. Seyd (Hrsg.), Große Transformation? Zur Zukunft moderner Gesellschaften (S. 3-33). Wiesbaden: Springer VS.

Dyson, E., Gilder, G., Keyworth, G., \& Toffler, A. (1994). Cyberspace and the American Dream: A Magna Carta for the Knowledge Age (22. Aug. 1994). http://www.pff.org/issues-pubs/futureinsights/fi1. 2magnacarta.html). Zugegriffen: März 2020.

Van Eeten, M. J. G., \& Mueller, M. (2012). Where is the governance in Internet governance? New Media \& Society, 15(5), 720-736.

Elder-Vass, D. (2016). Profit and gift in the digital economy. Cambridge: Cambridge University Press.

European Commission (Hrsg.). (2019). Antitrust: Commission fines Google $€ 1.49$ billion for abusive practices in online advertising. Press Release 20 March 2019. Brussels: European Commission. https:// ec.europa.eu/commission/presscorner/detail/en/IP_19_1770. Zugegriffen: März 2020.

Evans, D. S., \& Schmalensee, R. (2005). The industrial organization of markets with two-sided platforms. NBER Working Paper 11603. Cambridge, MA: National Bureau of Economic Research.

Evans, D. S., \& Schmalensee, R. (2016). Matchmakers. The new economics of multisided platforms. Boston: Harvard Business Review Press.

Feick, J., \& Werle, R. (2010). Regulation of cyberspace. In R. Baldwin, M. Cave \& M. Lodge (Hrsg.), The Oxford handbook of regulation (S. 523-547). Oxford: Oxford University Press.

Floyd, C. (1992). Software development as reality construction. In C. Floyd, H. Züllighoven, R. Budde \& R. Keil-Slawik (Hrsg.), Software development and reality construction (S. 86-100). Berlin: Springer.

Gerlitz, C., \& Helmond, A. (2013). The Like Economy: Social buttons and the data-intensive web. In New Media \& Society, 15(8), 1348-1365.

Gillespie, T. (2010). The politics of ,platforms“. New Media \& Society, 12(3), 347-364.

Gillespie, T. (2014). The relevance of algorithms. In T. Gillespie, P. Boczkowski \& K. Foot (Hrsg.), Media technologies. Essays on communication, materiality, and society (S. 167-194). Cambridge: MIT Press. 
Gillespie, T. (2016). Regulation of and by Platforms. In J. Burgess, T. Poell \& A. Marwick (Hrsg.), The SAGE Handbook of social media (S. 254-278). Los Angeles: Sage.

Gorwa, R. (2019). What is platform governance? Information, Communication \& Society, 22(6), 854-871.

Gorwa, R., Binns, R., \& Katzenbach, C. (2020). Algorithmic content moderation: Technical and political challenges in the automation of platform governance. Big Data \& Society, 7(1). https://doi.org/10. $1177 / 2053951719897945$.

Harris, B. (2020). Preparing the way forward for Facebook's Oversight Board. https://about.fb.com/news/ 2020/01/facebooks-oversight-board/. Zugegriffen: März 2020.

Haucap, J., \& Stühmeier, T. (2016). Competition and antitrust in Internet markets. In J. M. Bauer \& M. Latzer (Hrsg.), Economics of the Internet (S. 183-210). Cheltenham: Edward Elgar.

Helmond, A. (2015). The platformization of the web: Making web data platform ready. Social Media \& Society, 1(2), 1-11.

Hertwig, M., \& Papsdorf, C. (2017). Varieties of Sharing. Handlungsorientierung, Strukturen und Arbeitsbedingungen eines neuartigen Feldes. Berliner Journal für Soziologie, 27, 521-546.

International Monetary Fund (2018). Measuring the digital economy. Washington D.C.: IMF.

Just, N., \& Latzer, M. (2017). Governance by algorithms: Reality construction by algorithmic selection on the Internet. Media, Culture \& Society, 39(2), 238-258.

Katzenbach, C., \& Ulbricht, L. (2019). Algorithmic governance. Internet Policy Review, 8(4), 1-18.

Kenney, M., \& Zysman, J. (2016). The rise of the platform economy. Issues in Science and Technology, $32(3), 61-69$.

Khan, L. M. (2018). Amazon - an infrastructure service and its challenge to current antitrust law. In M. Moore \& M. Tambini (Hrsg.), Digital dominance. The power of Google, Amazon, Facebook, and Apple (S. 98-129). Oxford: Oxford University Press.

Kirchner, S., \& Beyer, J. (2016). Die Plattformlogik als digitale Marktordnung. Wie die Digitalisierung Kopplungen von Unternehmen löst und Märkte transformiert. Zeitschrift für Soziologie, 45(5), 324-339.

Kitchin, R. (2014). The data revolution. Big data, open data, data infrastructures \& their consequences. Los Angeles: Sage.

Kommission Wettbewerbsrecht 4.0 (2019). Ein neuer Wettbewerbsrahmen für die Digitalwirtschaft. Berlin: Bundesministerium für Wirtschaft und Energie.

Langley, P., \& Leyshon, A. (2016). Platform capitalism: The intermediation and capitalisation of digital economic circulation. Finance and Society, 2, 1-21.

Lessig, L. (1999). CODE and other laws of cyberspace. New York: Basic Books.

Libra Association (2019). An introduction to Libra. Whitepaper. https://libra.org/en-US/wp-content/ uploads/sites/23/2019/06/LibraWhitePaper_en_US.pdf. Zugegriffen: März 2020.

Lobigs, F., \& Neuberger, C. (2018). Meinungsmacht im Internet und die Digitalstrategien von Medienunternehmen. Leipzig: Vistas.

Mai, H. (2019). Libra - a global challenger in payments and for central banks? In J. Schildbach (Hrsg.), EU-Monitor - Digital economy and structural change. Frankfurt a. M.: Deutsche Bank Research.

Mazzucato, M. (2013). The entrepreneurial state. Debunking public vs. private sector myths. London: Anthem Press.

Misterek, F. (2017). Digitale Souveränität. Technikutopien und Gestaltungsansprüche demokratischer Politik. MPIfG Discussion Paper 17/11. Köln: MPIfG.

Nieborg, D. B., \& Helmond, A. (2019). The political economy of Facebook's platformization in the mobile ecosystem: Facebook Messenger as a platform instance. Media, Culture \& Society, 41(1), 196-218.

Nieborg, D. B., \& Poell, T. (2018). The platformization of cultural production: Theorizing the contingent cultural commodity. New Media \& Society, 20(11), 4275-4292.

Nielsen, R. K., \& Ganter, S. A. (2018). Dealing with digital intermediaries: A case study of the relations between publishers and platforms. New Media \& Society, 20(4), 1600-1617.

O'Reilly, T. (2005). What is Web 2.0. Design patterns and business models for the next generation of software. O'Reilly Network. http://oreilly.com/pub/a/web2/archive/what-is-web-20.html. Zugegriffen: März 2020.

Parker, G. G., Van Alstyne, M. W., \& Choudary, S. P. (2016). The Platform Revolution. New York: W.W. Norton.

Pasquale, F. (2015). The Black Box Society. The secret algorithms that control money and information. Cambridge: Harvard University Press.

Rahman, K. S., \& Thelen, K. (2019). The rise of the platform business model and the transformation of twenty-first century capitalism. Politics \& Society, 47(2), 177-204. 
Reich, R. (2018). Break up Facebook (and while we're at it, Google, Apple and Amazon). The Guardian, 20.11.2018.

Rochet, J.-C., \& Tirole, J. (2003). Platform competition in two-sided markets. Journal of the European Economic Association, 1(4), 990-1029.

Schmeling, M. (2019). What is Libra? Understanding Facebooks currency. SAFE Policy Letter, No. 76. Frankfurt a. M.: Goethe University Frankfurt.

Schrape, J.-F. (2019). The promise of technological decentralization. A brief reconstruction. Society, 56(1), 31-38.

Schulz, W. (2018). Regulating intermediaries to protect privacy online - the case of the German NetzDG. HIIG Discussion Paper Series 2018-01. Berlin: HIIG.

Srnicek, N. (2017). Platform Capitalism. Cambridge: Polity.

Staab, P. (2019). Digitaler Kapitalismus. Markt und Herrschaft in der Ökonomie der Unknappheit. Berlin: Suhrkamp.

Sundararajan, A. (2016). The sharing economy. The end of employment and the rise of crowd-based capitalism. Cambridge: MIT Press.

Taskinsoy, J. (2019). Facebook's Project Libra: Will Libra sputter out or spur central banks to introduce their own unique cryptocurrency projects? Sarawak: University Malaysia Sarawak.

Thelen, K. (2018). Regulating Uber: The politics of the platform economy in Europe and the United States. Perspectives on Politics, 16(4), 938-953.

Viscusi, W.K., Harrington Jr., J. E., \& Sappington, D.E.M. (2018). Economics of regulation and antitrust. Fifth Edition. Cambridge: MIT Press.

Voß, G. G. (2020). Der arbeitende Nutzer. Über den Rohstoff des Überwachungskapitalismus. Frankfurt a. M.: Campus.

Winner, L. (1980). Do artifacts have politics? Daedalus, 109(1), 121-136.

Yeung, K. (2018). Algorithmic regulation: A critical interrogation. Regulation \& Governance, 12(4), $505-523$.

Zerdick, A., Schrape, K., Artope, A., Goldhammer, K., Heger, D.K., Lange, U., Vierkant, E., Lopez-Escobar, E., \& Silverstone, R. (2001). Die Internet-Ökonomie. Strategien für die digitale Wirtschaft. Berlin: Springer.

Zuboff, S. (2019). The age of surveillance capitalism: The fight for the future at the new frontier of power. London: Profile Books.

Zysman, J., \& Kenney, M. (2016). The next phase in the digital revolution. Platforms, abundant computing, growth and employment. ETLA Reports No. 61. Helsinki: The Research Institute of the Finnish Economy.

Ulrich Dolata geb. 1959. Dr. rer. pol. habil., Professor für Organisations- und Innovationssoziologie an der Universität Stuttgart und Geschäftsführender Direktor des Instituts für Sozialwissenschaften. Arbeitsschwerpunkte: Technik- und Innovationsforschung, organisationaler und institutioneller Wandel, politische Ökonomie des Internets. Ausgewählte Veröffentlichungen: The transformative capacity of new technologies. A theory of sociotechnical change, 2013; (mit J.-F. Schrape) Collectivity and power on the Internet. A sociological perspective, 2018; Technisch erweiterte Sozialität. Soziale Bewegungen und das Internet, in: Zeitschrift für Soziologie, 2017; Volatile Monopole. Konzentration, Konkurrenz und Innovationsstrategien der Internetkonzerne, in: Berliner Journal für Soziologie, 2015. 